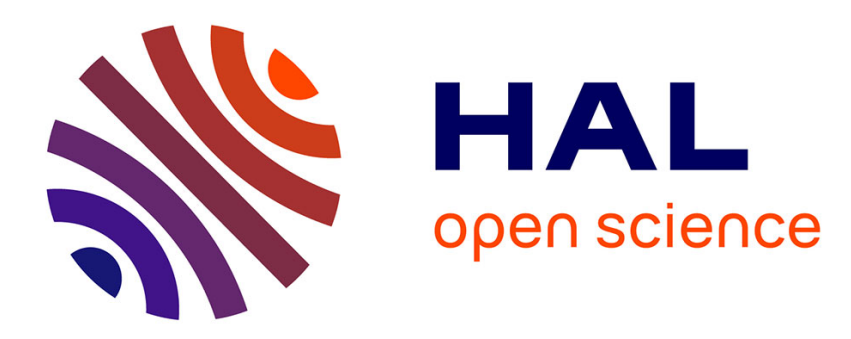

\title{
On the kinematic approximation to reflectivity
}

Devinderjit Singh Sivia

\section{To cite this version:}

Devinderjit Singh Sivia. On the kinematic approximation to reflectivity. Philosophical Magazine, 2007, 87 (10), pp.1575-1580. 10.1080/14786430601072283 . hal-00513803

\section{HAL Id: hal-00513803 \\ https://hal.science/hal-00513803}

Submitted on 1 Sep 2010

HAL is a multi-disciplinary open access archive for the deposit and dissemination of scientific research documents, whether they are published or not. The documents may come from teaching and research institutions in France or abroad, or from public or private research centers.
L'archive ouverte pluridisciplinaire HAL, est destinée au dépôt et à la diffusion de documents scientifiques de niveau recherche, publiés ou non, émanant des établissements d'enseignement et de recherche français ou étrangers, des laboratoires publics ou privés. 


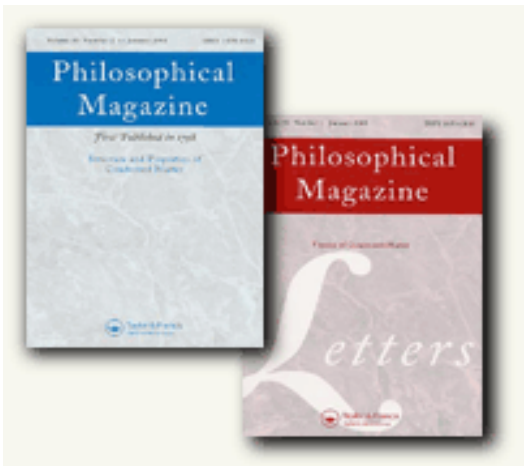

\section{On the kinematic approximation to reflectivity}

\begin{tabular}{|r|l|}
\hline Journal: & Philosophical Magazine \& Philosophical Magazine Letters \\
\hline Manuscript ID: & TPHM-06-Jul-0254 \\
\hline Journal Selection: & Philosophical Magazine \\
\hline Date Submitted by the & $14-J u l-2006$ \\
\hline Keywords: & $\begin{array}{l}\text { reflectivity, neutron diffraction, neutron scattering, X-ray } \\
\text { diffraction, X-ray scattering }\end{array}$ \\
\hline Keywords (user supplied): & \\
\hline Nist of Authors: & Sivia, Devinderjit; Rutherford Appleton Laboratory, ISIS facility \\
\hline $\begin{array}{l}\text { Note: The following files were submitted by the author for peer review, but cannot be converted } \\
\text { to PDF. You must view these files (e.g. movies) online. }\end{array}$ \\
\hline Sivia 06_Jul_0254 181006.tex
\end{tabular}

\section{(s) ScholaroNE \\ Manuscript Central}


Philosophical Magazine,

Vol. 00, No. 00, DD Month 200x, 1-5

\title{
1 Introduction
}

\begin{abstract}
The kinematic approximation to specular reflectivity is very useful for understanding neutron and X-ray scattering observations, because it provides a simple analytical formula that relates the scattering-length density profile of a layered surface or interface, $\beta(z)$, to the data, $R\left(q_{z}\right)$. Although its derivation from the wave equation, or the optical transfer model, for large $q_{z}$ is straightforward, the origin of the $q_{z}$-factor in the denominator is less obvious when the problem is viewed in the limit of Fraunhofer diffraction from the outset. This paper, which is principally educational, aims to bring clarity to the issue.
\end{abstract}

The opportunities for doing experiments at neutron and synchrotron facilities, to study matter at the atomic and molecular level, have grown rapidly in recent years. This has been accompanied by a shift in the demographics of the users, from principally physicists to a broad range of natural scientists. Given such an increasingly diverse spread in the physics backgrounds and mathematical abilities of new graduate students entering the field, what is the best way of introducing them to the elementary theory of scattering experiments?

A valuable approach is to motivate the general result for the (Born-Fraunhofer) differential cross-section, $\mathrm{d} \sigma / \mathrm{d} \Omega$, following Lovesey [1] and Squires [2],

$$
\frac{\mathrm{d} \sigma}{\mathrm{d} \Omega}=\left|\iiint \beta(\boldsymbol{r}) \mathrm{e}^{-\mathrm{i} \boldsymbol{r} \bullet \boldsymbol{q}} \mathrm{d}^{3} \boldsymbol{r}\right|^{2} \propto S(\boldsymbol{q}),
$$

where $\beta$ is the scattering-length density of the sample as a function of position $\boldsymbol{r}, \boldsymbol{q}$ is the wave-vector transfer of the neutron or X-ray and $\mathrm{i}^{2}=-1$, and to explain how the elastic scattering law, $S(\boldsymbol{q})$, can be understood qualitatively with a basic understanding of Fourier transforms. Although inelastic processes can be accommodated in this formalism, through the temporal extension of equation (1) for the partial differential cross-section $[1,2]$, the central message of this approach is appreciated most easily by considering one-dimensional examples of equation (1). Specular reflectivity from (perfectly) layered surfaces or interfaces is a prime example, therefore, which allows data from common structures to be interpreted readily by analogy with elementary diffraction patterns familiar from high school.

The kinematic approximation to the specular reflectivity, $R\left(q_{z}\right)$, which is applicable for weak scattering in the far-field limit, is given by [3]

$$
R\left(q_{z}\right)=\frac{16 \pi^{2}}{q_{z}^{2}}\left|\int \beta(z) \mathrm{e}^{-\mathrm{i} z q_{z}} \mathrm{~d} z\right|^{2}
$$

where $z$ is the direction perpendicular to the layers of the sample, as illustrated in figure 1 , and $q_{z}$ is the corresponding component of the wave-vector transfer; it works well for large $q_{z}$, typically when $q_{z}^{2} \gg 16 \pi\left|\beta_{s}\right|$ where $\beta_{s}$ is the scattering-length density of the substrate. While it seems immediately plausible that the Fourier part of equation (2) is related to equation (1), for the case where the sample is invariant in the

\footnotetext{
*Email: dss@isise.rl.ac.uk
} 


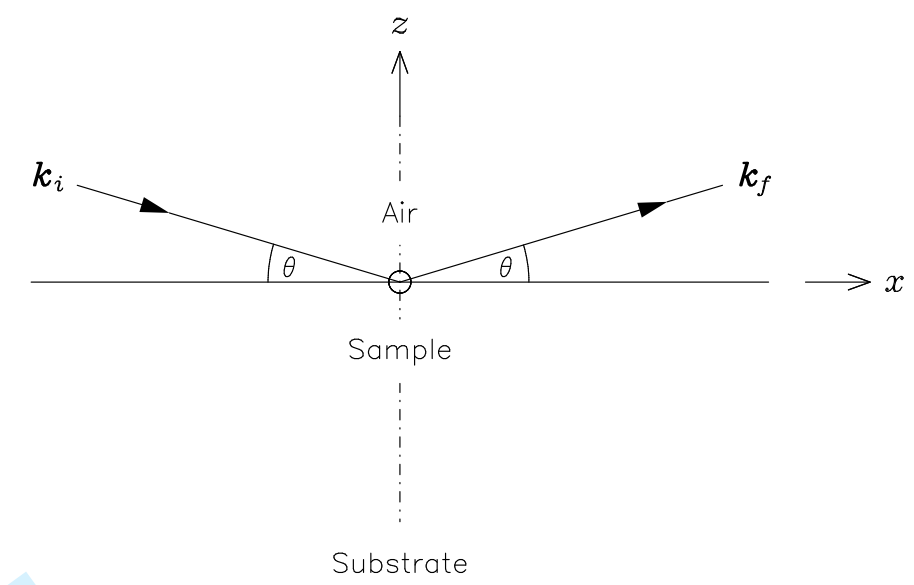

Figure 1. A schematic illustration of the setup for specular reflectivity.

$x-y$ plane, the origin of the prefactor is less obvious. Although the $q_{z}^{2}$ in the denominator makes sense on dimensional grounds, how does it arise physically?

The search for a simple answer to the above question, that many would find both intuitive and plausible, led to numerous discussions with colleagues and the consultation of authoritative books and papers [1-10]. The analysis given below is the result of that investigation. Although it has much in common with the more standard formulation of Pynn [7], it adopts a very different approach to the evaluation of a crucial integral. This alternative derivation makes every step of the calculation completely transparent, and provides greater physical insight.

\section{The differential cross-section}

Let us begin by formally defining the basic characteristics of the samples under consideration. In the context of figure 1, they have the property that their scattering-length density varies only in the $z$ direction:

$$
\beta(\boldsymbol{r})=\left\{\begin{array}{cl}
\beta(z) & \text { for }|x|<L_{x} \text { and }|y|<L_{y} \\
0 & \text { otherwise }
\end{array}\right.
$$

where we have taken the finite illumination of the $x-y$ plane to be over a rectangular region, $|x|<L_{x}$ and $|y|<L_{y}$, for simplicity. The substitution of $\beta(\boldsymbol{r})$ from equation (3) into equation (1), with $\boldsymbol{r}=(x, y, z)$ and wave-vector transfer

$$
\boldsymbol{q}=\left(q_{x}, q_{y}, q_{z}\right)=\boldsymbol{k}_{i}-\boldsymbol{k}_{f}
$$

where $\hbar \boldsymbol{k}_{i}$ and $\hbar \boldsymbol{k}_{f}$ are the momenta of the incident and scattered particles respectively, leads to the decomposition of the differential cross-section into a product of three terms:

$$
\frac{\mathrm{d} \sigma}{\mathrm{d} \Omega}=\left|\int_{-L_{x}}^{L_{x}} \mathrm{e}^{-\mathrm{i} x q_{x}} \mathrm{~d} x\right|^{2}\left|\int_{-L_{y}}^{L_{y}} \mathrm{e}^{-\mathrm{i} y q_{y}} \mathrm{~d} y\right|^{2}\left|\int \beta(z) \mathrm{e}^{-\mathrm{i} z q_{z}} \mathrm{~d} z\right|^{2}
$$

where we have omitted the limits on $z$-integral, as being irrelevant to the present argument (but would be $-\infty$ and zero, with $\beta_{A i r}=0$ ). The $x$ and $y$ integrals are readily evaluated to yield the classical sinc functions,

$$
\int_{-L}^{L} \mathrm{e}^{-\mathrm{i} t q} \mathrm{~d} t=\left[\frac{\mathrm{e}^{-\mathrm{i} t q}}{-\mathrm{i} q}\right]_{-L}^{L}=\frac{2}{q}\left[\frac{\mathrm{e}^{\mathrm{i} L q}-\mathrm{e}^{-\mathrm{i} L q}}{2 \mathrm{i}}\right]=\frac{2 \sin (L q)}{q}
$$




\section{Page 3 of 7}

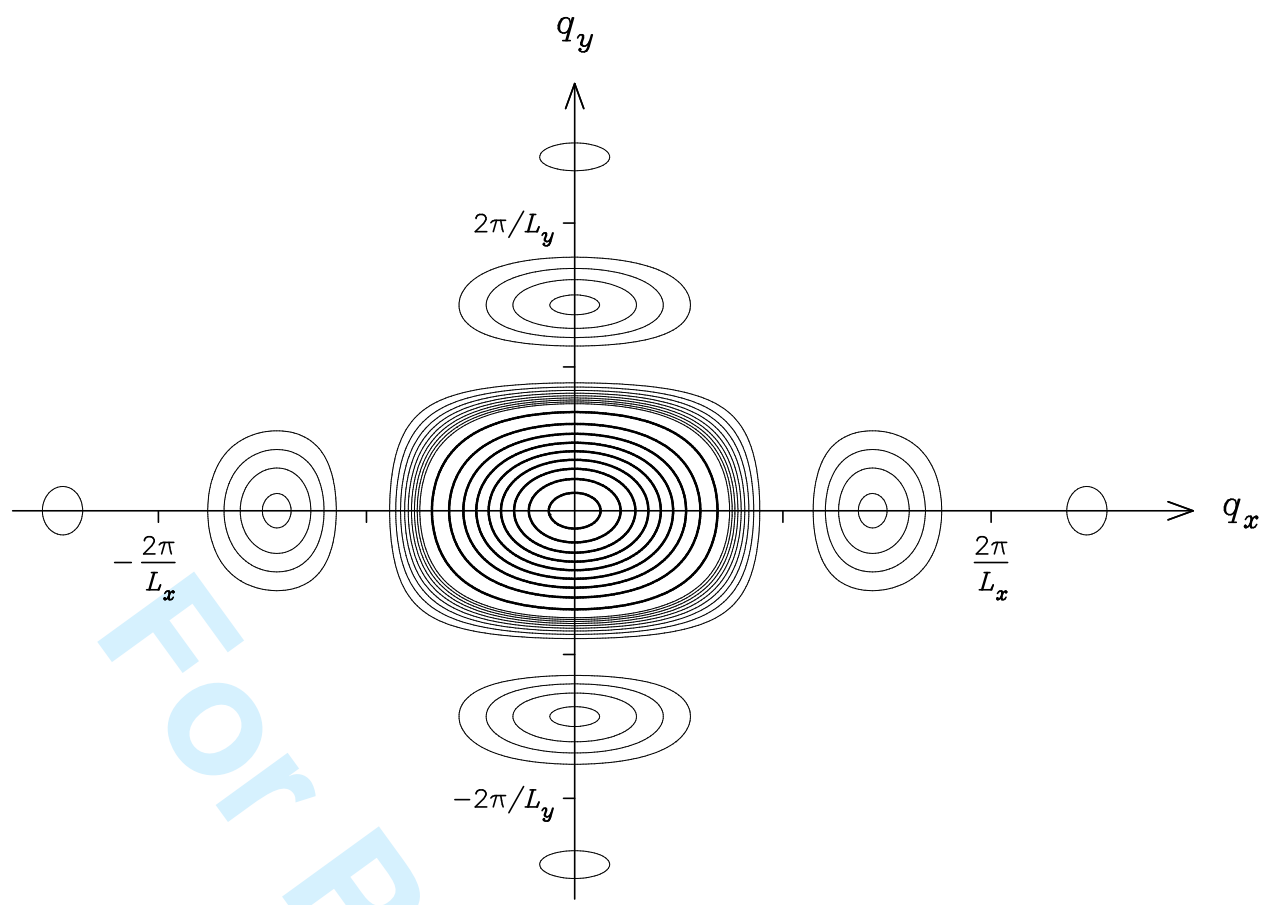

Figure 2. The $\left(q_{x}, q_{y}\right)$-dependent sinc-squared factor in equation (7), contoured linearly: the highest values, from 0.15 to 0.95 of the maximum, are shown with a thick line whereas lower ones (0.015 to 0.095) appear thin.

so that, as seen from figure 2 , the differential cross-section is sharply-peaked around $q_{x}=0$ and $q_{y}=0$ :

$$
\frac{\mathrm{d} \sigma}{\mathrm{d} \Omega}=16 \frac{\sin ^{2}\left(L_{x} q_{x}\right)}{q_{x}^{2}} \frac{\sin ^{2}\left(L_{y} q_{y}\right)}{q_{y}^{2}}\left|\int \beta(z) \mathrm{e}^{-\mathrm{i} z q_{z}} \mathrm{~d} z\right|^{2} .
$$

\section{Reflectivity and the differential cross-section}

To relate the differential cross-section to the reflectivity, we need to remind ourselves of their exact definitions. The former tells us the rate at which neutrons are scattered with wave-vector transfer $\boldsymbol{q}$, per unit solid angle, compared to the rate at which they are incident on the sample, per unit area perpendicular to the incoming beam:

$$
\frac{\mathrm{d} \sigma}{\mathrm{d} \Omega}=\frac{\text { Rate of scattering in direction } \boldsymbol{q} \text { per unit solid angle }}{\text { Rate of incidence per unit area } \perp \text { to incoming beam }} .
$$

The reflectivity is the proportion of incident neutrons that change direction normal to the surface of the sample upon scattering:

$$
R=\frac{\text { Rate of reflective-scattering }}{\text { Rate of incidence }}
$$

The denominators of equations (8) and (9) are related through the area of the sample illuminated perpendicular to the incoming beam, $4 L_{x} L_{y} \sin \theta$, whereas the numerator of $\mathrm{d} \sigma / \mathrm{d} \Omega$ has to be integrated over the solid angle corresponding to neutrons 'bouncing back' to yield $R$. Since the latter calculation is a bit awkward, let us make use of the physical insight provided by the fact that the differential cross-section of equation (7) is sharply-peaked, around $q_{x}=0$ and $q_{y}=0$, to approximate the required integral by

$$
\left.\iint \frac{\mathrm{d} \sigma}{\mathrm{d} \Omega} \mathrm{d} \Omega \approx \frac{\mathrm{d} \sigma}{\mathrm{d} \Omega}\right|_{q_{x}=0, q_{y}=0} \Delta \Omega
$$




\section{Philosophical Magazine \& Philosophical Magazine Letters}

where $\Delta \Omega$ is the solid angle spread of the scattered beam around the outgoing vector $\boldsymbol{k}_{f}$. Hence, the substitution of $\mathrm{d} \sigma / \mathrm{d} \Omega$ from equation (7) into the right-hand side of equation (10), and its division by the illuminated area, allows the reflectivity to be written as

$$
R \approx 16 L_{x}^{2} L_{y}^{2}\left|\int \beta(z) \mathrm{e}^{-\mathrm{i} z q_{z}} \mathrm{~d} z\right|^{2} \frac{\Delta \Omega}{4 L_{x} L_{y} \sin \theta}
$$

\section{The divergence of the reflected beam}

To complete the calculation for the kinematic approximation to specular reflectivity, we need an estimate for $\Delta \Omega$. This can be obtained by considering an incoming beam which is parallel to the $x$-axis, so that the $y$-component of $\boldsymbol{k}_{i}$ is zero (as in figure 1),

$$
\boldsymbol{k}_{i}=\left(k_{x}, 0, k_{z}\right)
$$

a reflected beam with wave-vector

$$
\boldsymbol{k}_{f}=\left(k_{x}+\epsilon, \delta, \xi-k_{z}\right)
$$

where $\epsilon, \delta$ and $\xi$ are parameters that denote the departure from 'perfect' reflection, and the requirement that kinetic energy be conserved in elastic scattering, $\left|\boldsymbol{k}_{i}\right|^{2}=\left|\boldsymbol{k}_{f}\right|^{2}$, which imposes the condition that

$$
\epsilon^{2}+2 \epsilon k_{x}+\delta^{2}+\xi^{2}-2 \xi k_{z}=0 .
$$

Since we are only interested in the neighbourhood of $q_{x}=0$ and $q_{y}=0$, or equivalently $\epsilon \approx 0$ and $\delta \approx 0$, the first-order terms in equation (14) yield a linear correlation between the $x$ and $z$ components of the wave-vector transfer:

$$
\xi \approx\left[\frac{k_{x}}{k_{z}}\right] \epsilon
$$

This means that the unit vector along the scattered beam, $\widehat{\boldsymbol{k}}_{f}$, is a function of the independent variables $\epsilon$ and $\delta$ :

$$
\widehat{\boldsymbol{k}}_{f}(\epsilon, \delta) \approx \frac{\left(k_{x}+\epsilon, \delta,\left[\frac{k_{x}}{k_{z}}\right] \epsilon-k_{z}\right)}{\sqrt{k_{x}^{2}+k_{z}^{2}}}
$$

Given equation (16), and the nature of the sinc functions in equation (7) and figure 2, we can estimate the solid angle spread of the reflected beam from the area of the parallelogram subtended by the vertex of $\widehat{\boldsymbol{k}}_{f}$ when pointing in the directions defined by $\epsilon \approx \pm \pi / 2 L_{x}$ and $\delta \approx \pm \pi / 2 L_{y}$ :

$$
\Delta \Omega \approx\left|\left[\widehat{\boldsymbol{k}}_{f}\left(\frac{\pi}{2 L_{x}}, 0\right)-\widehat{\boldsymbol{k}}_{f}\left(\frac{-\pi}{2 L_{x}}, 0\right)\right] \times\left[\widehat{\boldsymbol{k}}_{f}\left(0, \frac{\pi}{2 L_{y}}\right)-\widehat{\boldsymbol{k}}_{f}\left(0, \frac{-\pi}{2 L_{y}}\right)\right]\right|
$$

After the substitution for the four $\widehat{\boldsymbol{k}}_{f}$ 's from equation (16),

$$
\Delta \Omega \approx \frac{1}{k_{x}^{2}+k_{z}^{2}}\left|\left(\frac{\pi}{L_{x}}, 0, \frac{k_{x}}{k_{z}} \frac{\pi}{L_{x}}\right) \times\left(0, \frac{\pi}{L_{y}}, 0\right)\right|
$$


and the evaluation of the cross-product,

$$
\Delta \Omega \approx \frac{\pi^{2}}{L_{x} L_{y}\left(k_{x}^{2}+k_{z}^{2}\right)}\left|\left(-\frac{k_{x}}{k_{z}}, 0,1\right)\right|,
$$

a rearrangement of the modulus allows the solid angle to be written as

$$
\Delta \Omega \approx \frac{\pi^{2} \sin \theta}{L_{x} L_{y} k_{z}^{2}}
$$

where $\sin \theta=k_{z} / \sqrt{k_{x}^{2}+k_{z}^{2}}$ from figure 1 and elementary trigonometry. Finally, the substitution of $\Delta \Omega$ from equation (20) into equation (11), using the principal wave-vector transfer $q_{z}=-2 k_{z}$, leads to the desired reflectivity formula of equation (2).

\section{Conclusions}

We have derived the formula for the kinematic approximation to specular reflectivity directly from the general expression of equation (1) for weak scattering in the far-field limit. Every step of the calculation has been made transparent to reveal the origin of the $q_{z}^{2}$-term in the denominator of equation (2): it arises from the divergence of the reflected beam, whose angular spread is proportional to the square of the wavelength of the neutron or X-ray photon. Explicitly, with $k_{z}=(2 \pi / \lambda) \sin \theta$, equation (20) tells us that $\Delta \Omega \propto \lambda^{2} / \sin \theta$.

When presented with the above conclusion, many are initially sceptical about the spreading out of the reflected beam and its role in the origin of the $q_{z}^{2}$-term (although most eventually come around to it). Comments about it being an effect due to the 'finite size of the sample' are sometimes made in passing, although the significance is usually left unclear. While it is true that the absolute magnitude of the solid angle spread increases as the illuminated area of the sample decreases, as can be seen from equation (20), it is the relative values of $\Delta \Omega$ for a given $L_{x}$ and $L_{y}$ (and $\theta$ ) that counts; that depends on the square of the wavelength, and is a result that holds even as $L_{x} \rightarrow \infty$ and $L_{y} \rightarrow \infty$.

\section{Acknowledgments}

I am grateful to Rob Dalgliesh, Nikitas Gidopoulos, Bill Hamilton, Stephen Holt, Sean Langridge, Stephen Lovesey, Jerry Mayers, Jeff Penfold, Roger Pynn, Jorge Quintanilla, Rob Richardson, Greg Smith and John Webster for useful discussions.

\section{References}

[1] S. W. Lovesey, Theory of Neutron Scattering from Condensed Matter (Oxford University Press, Oxford, 1984).

[2] G. L. Squires, Introduction to the Theory of Thermal Neutron Scattering (Cambridge University Press, Cambridge, 1978).

[3] T. L. Crowley, E. M. Lee, E. A. Simister, R. K. Thomas, J. Penfold and A. R. Rennie, Colloids and Surfaces 5285 (1990).

[4] J. Penfold and R. K. Thomas, J. Phys.-Condens. Matt. 61369 (1990).

[5] T. P. Russell, Ann. Rev. Mat. Sci. 21249 (1991).

[6] R. Pynn, Acta Cryst. B 312555 (1975).

[7] R. Pynn, http://www.mrl.ucsb.edu/ pynn/Lecture_4_Reflectivity.pdf.

[8] J. Als-Nielsen, Z. Phys. B - Condensed Matter 61411 (1985).

[9] A. G. Klein and S. A. Werner, Rep. Prog. Phys. 46259 (1983).

[10] M. Born and E. Wolf, Principles of Optics (Pergamon Press, London and New York, 1959). 


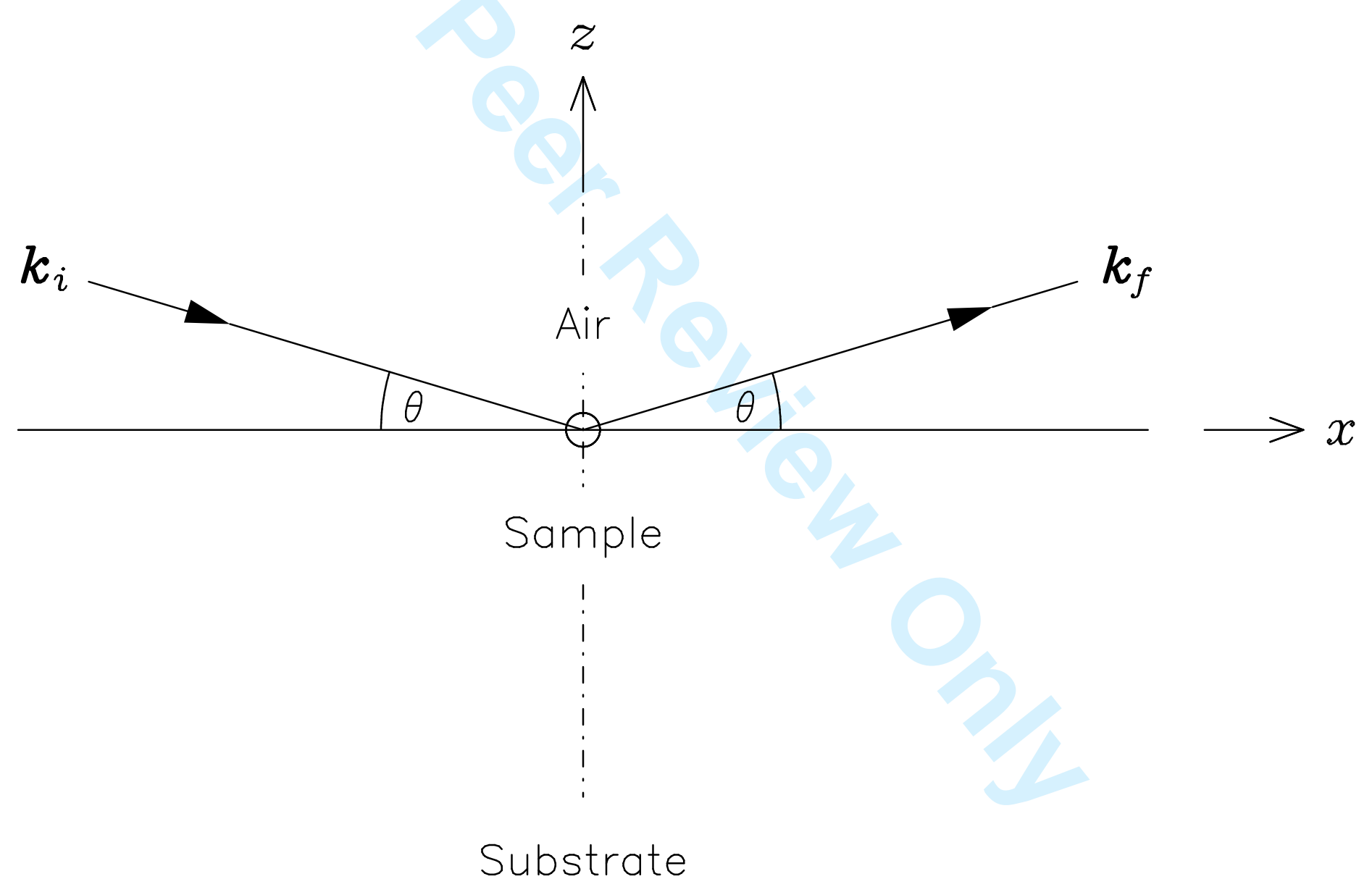



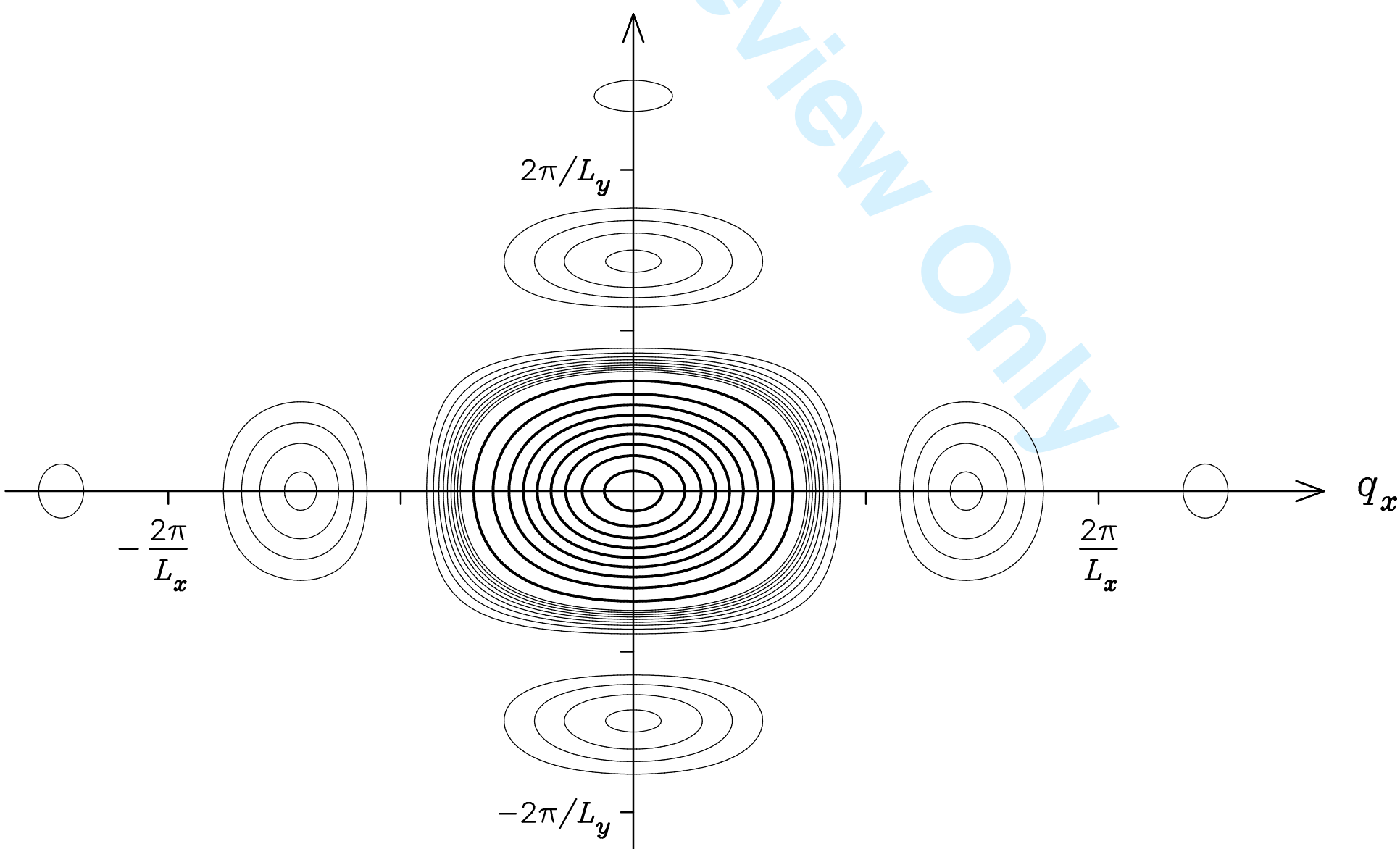

http://mc. nanjuscriptcentral.com/pm-pml 